\title{
Role of postgraduate researchers in advancing cancer and biomedical research
}

\author{
Saly F. Gheda \\ Department of Botany and Microbiology, Faculty of Science, Tanta University, Egypt
}

\section{ABSTRACT}

This special issue of IJCBR "Role of postgraduate researchers in advancing cancer and biomedical research" aims to raise awareness of the importance of postgraduate scientific research in the fields of cancer and biomedical research among scholars and junior researchers. There is always a need to discover safe natural materials or novel synthetic compounds to treat different types of cancer as well as other diseases. The master and PhD theses' abstracts presented in this special issue cover six topics, including Anticancer therapeutics, Cancer biology and immunology, Drug discovery, Biological activities of biochemicals, Nanotechnology, and Medicine and Nursing.

In Anticancer therapeutics section, several synthetic compounds as well as extracts from marine organisms and plants showed effective anti-cancer cell capabilities., opening new avenues for their use a possible adjuvant to cancer therapy. In Cancer Biology and Immunology section, the studies have shown some intrinsic and extrinsic features of cancer cells as well as the potential as cancer immunotherapeutic. In Drug Discovery Section, certain materials extracted from micro and marine algae, and probiotic bacteria showed as anti-diabetic and antibacterial, and anti-schistosomiasis, as well as stimulators for immune response against hepatitis $\mathrm{C}$ Virus and as a flavoring agent in some foods. In Biological Activities of Biochemicals section, synthetic chemicals such as heterocyclic compounds, bicyclic, and polycyclic rings demonstrated potential effects as antimicrobials, anti-oxidants, and anti-tumor capabilities. In Nanotechnology section, green metal nanoparticles biosynthesized using Moringa oleifera plants and blue green algae expressed anti-microbial, anti-oxidant, anti-viral, and anti-cancer properties. In Medicine and Nursing, the studies recommended that nurses should be educated with programs such as burn, PICC line in order to develop their awareness and practice.

Overall, the studies presented several natural and chemical compounds with different biological activities, including anti-diabetic, anti-microbial, anti-cancer, as well as immune enhancer, opening new avenue for drug discovery. I do believe that the scientific findings from the presented theses are of great importance and add values for the biomedical research in general and cancer research in particular. These studies highlight the fundamental role of postgraduate students in advancing biomedical research and innovation.

Editor-in-Chief: Prof. M.L. Salem, PhD - Article DOI: 10.21608/JCBR.2021.75093.1210 